International Journal of Pure and Applied Mathematics

Volume 106 No. 3 2016, 957-964

ISSN: 1311-8080 (printed version); ISSN: 1314-3395 (on-line version)

url: http://www.ijpam.eu

doi: 10.12732/ijpam.v106i3.19

\title{
A FIXED POINT THEOREM FOR VOLUME PRESERVING LINEAR TRANSFORMATIONS
}

\author{
Martin Moskowitz \\ Ph.D. Program in Mathematics \\ City University of New York Graduate Center \\ 365 Fifth Ave, New York, NY 10016-4309, USA
}

Abstract: In this note we derive consequences of the fact that if $g \in \operatorname{SL}(n)$, where $n \geq 2$, and $\sigma_{i}(g)$ are the coefficients of its characteristic polynomial, then $g$ has 1 as an eigenvalue if and only if $\sum_{i=1}^{n-1}(-1)^{i-1} \sigma_{i}(g)=0$ or 2 according to the parity of $n$. These are:

Corollary 2. Let $D$ be a real or complex $n \times n$ matrix of $\operatorname{tr}(D)=0$. If $D$ is singular, then one of the following equations holds (according to the parity of $n$ ).

$$
\sum_{i=1}^{n-1}(-1)^{i-1} \sigma_{i}(\operatorname{Exp} D)=0 \quad \sum_{i=1}^{n-1}(-1)^{i-1} \sigma_{i}(\operatorname{Exp} D)=2 .
$$

Conversely, if $D$ has no eigenvalues in $2 \pi i \mathbb{Z}$ and the appropriate one of these equations holds, $D$ must be singular.

Corollary 3. Let $A \in M_{n}(\mathbb{R})=\mathcal{M}$ which acts on $\mathcal{M}$ by $\operatorname{ad}_{A}(X)=[A, X]$. If $A$ has only non-negative eigenvalues and one of the following equations holds (according to the parity of n)

$$
\sum_{i=1}^{n^{2}-1}(-1)^{i-1} \sigma_{i}\left(\operatorname{Ad}_{\operatorname{Exp}(A)}\right)=0 \quad \sum_{i=1}^{n^{2}-1}(-1)^{i-1} \sigma_{i}\left(\operatorname{Ad}_{\operatorname{Exp}(A)}\right)=2,
$$

then $[A, X]=0$ for some $X \in \mathcal{M}$, not a linear combination of $A$ and $I$.

Corollary 4. Let $A \in \mathcal{M}$ and have all non-negative eigenvalues. Then the action of $\operatorname{Exp}(A)$ on $\mathcal{M}$ by conjugation has a fixed point which is not a linear combination of $A$ and $I$.

AMS Subject Classification: 15A04, 15A16, 15A24, 15A27, 17B40, 20G20, 22E15

Key Words: fixed point, eigenvalue, determinant, trace, exponential map, singular matrix, adjoint action, Lie algebra, centralizer

Received: December 17, 2015

Published: March 4, 2016 (c) 2016 Academic Publications, Ltd. url: www.acadpubl.eu 
Let $K$ be a field, $g \in \mathrm{GL}(n, K)$, where $n \geq 2$ and

$$
\chi_{g}(x)=x^{n}-\sigma_{1}(g) x^{n-1}+\sigma_{2}(g) x^{n-2}-\ldots+(-1)^{n} \operatorname{det}(g)
$$

be its characteristic polynomial. Since $g$ is invertible, it not only acts on $K^{n}$, but also on $K^{n} \backslash(0)$. Here we shall be interested in when $g$ has a fixed point under this action, or equivalently when $g$ has 1 as an eigenvalue. We consider the map GL $(n, K) \rightarrow K^{n-1}$ given by $g \mapsto\left(\sigma_{1}(g), \ldots, \sigma_{n-1}(g)\right)$. For this to occur the image of $g$ under this map must lie in a certain hyperplane and conversely. We consider the following equation.

$$
\sum_{i=1}^{n-1}(-1)^{i-1} \sigma_{i}(g)=1+(-1)^{n} \operatorname{det}(g) .
$$

When $g \in \mathrm{SL}(n, K)$ equation 1 becomes

$$
\sum_{i=1}^{n-1}(-1)^{i-1} \sigma_{i}(g)=1+(-1)^{n} .
$$

Theorem 1. Let $g \in \mathrm{GL}(n, K)$. Equation 1 holds, if and only if $g$ has a fixed point in this action. (Here the parity of $n$ doesn't matter.) Hence when $g \in \mathrm{SL}(n, K), g$ has a fixed point if and only if equation 2 holds. That is, $\sum_{i=1}^{n-1}(-1)^{i-1} \sigma_{i}(g)=0$ or 2 according to the parity of $n$.

Proof. If $g \in \mathrm{GL}(n, K)$ and the equation holds, then evidently $\chi_{g}(1)=0$ so 1 is an eigenvalue and conversely.

When $g \in \mathrm{SL}(n, K)$ this result seems remarkably similar to the fact that the Euler characteristic, $\chi\left(S^{n}\right)$, of the $n$ sphere is $1+(-1)^{n}$ expressing the fact that the Euler characteristic based on chains is the same as that based on homology. We illustrate it with some examples.

- Let $g \in \mathrm{SL}(2, K)$, where $K=\mathbb{R}$ or $\mathbb{C}$ acting as usual on $K^{2}$. Then $g$ has a fixed point if and only if $\operatorname{tr}(g)=2$. This means $g$ has 1 as an eigenvalue with multiplicity 2 and so is conjugate to a unitriangular matrix and conversely.

- Let $G=\mathrm{SO}(3, \mathbb{R})$. By Euler's theorem every $g \in G$ has a rotation axis and eigenvalue 1. Hence $\operatorname{tr}(g)=\sigma_{2}(g)$ for all $g \in G$. 
- Let $A=\left(a_{i, j}\right)$ be a stochastic matrix of order $n$ (all $a_{i, j} \geq 0$ and $\sum_{j} a_{i, j}=$ 1 for all $i$ ). Then the theorem of Perron-Frobenius (see [2] pg. 17-18) tells us, among other things, that $A$ has 1 as an eigenvalue. Hence equation 1 holds (whether $A$ is invertible, or not).

- One can construct a large number of matrices in $\operatorname{SL}(3, \mathbb{Z})$ all of which have 1 as an eigenvalue. For $g \in \mathrm{SL}(3, \mathbb{Z}), \chi_{g}(x)$ has its coefficients in $\mathbb{Z}$ and, 1 is an eigenvalue if and only if $\operatorname{tr}(g)=\sigma_{2}(g)$. Since, as we shall see below, $\sigma_{2}(g) \neq 0$, this means $\frac{\operatorname{tr}(g)}{\sigma_{2}(g)}=1$. Let

$$
g=\left[\begin{array}{ccc}
0 & 0 & 1 \\
1 & m & p \\
0 & 1 & n
\end{array}\right]
$$

where $n, m$ and $p$ are integers. Then $\chi_{g}(x)=x^{3}-(n+m) x^{2}+(m n-p) x-1$. Thus $\operatorname{det}(g)=1, \operatorname{tr}(g)=n+m$ and $\sigma_{2}(g)=m n-p$. We consider Pythagorean triples of integers, $r^{2}=s^{2}+t^{2}$, and their multiples by $\nu=$ $1,2, \ldots, \nu^{2} r^{2}=\nu^{2} s^{2}+\nu^{2} t^{2}$. Let $r$ and $\nu$ be chosen so that $\operatorname{tr}(g)=2 \nu r$ and $\sigma_{2}(g)=\nu^{2} s^{2}-p$. We choose $p$ so that $\operatorname{tr}(g) / \sigma_{2}(g)=1$. Since $\frac{\operatorname{tr}(g)}{\sigma_{2}(g)}=\frac{2 \nu r}{\nu^{2} s^{2}-p}$, this will be 1 exactly when $p=\nu^{2} s^{2}-2 \nu r$. This determines the integer $p$. Hence $2 \nu r=\sigma_{2}(A)=m n-p=m n-\left(\nu^{2} s^{2}-2 \nu r\right)$. Hence $m n=\nu^{2} s^{2}=\nu^{2} r^{2}-\nu^{2} t^{2}=(\nu r+\nu t)(\nu r-\nu t)$. Taking $m=\nu r+\nu t$ and $n=\nu r-\nu t$ (or vice versa), $g$ has entries $m=\nu r+\nu t \quad n=$ $\nu r-\nu t \quad p=\nu^{2} s^{2}-2 \nu r$ (and $\operatorname{tr}(A)=m+n=2 \nu r$ ). Thus for each multiple of a Pythagorean triple we have a solution and there are infinitely many Pythagorean triples as well.

Our theorem has the following corollary:

Corollary 2. Let $D$ be a real or complex $n \times n$ matrix of $\operatorname{tr}(D)=0$. If $D$ is singular, then one of the following equations holds (depending on whether $n$ is odd or even).

$$
\sum_{i=1}^{n-1}(-1)^{i-1} \sigma_{i}(\operatorname{Exp} D)=0 \quad \sum_{i=1}^{n-1}(-1)^{i-1} \sigma_{i}(\operatorname{Exp} D)=2 .
$$

Conversely, if $D$ has no eigenvalues in $2 \pi i \mathbb{Z}$ and the appropriate one of these equations holds, $D$ must be singular. 
In particular, suppose $\mathfrak{g}$ is a real or complex Lie algebra and $D$ is a derivation of trace 0 . Let $\alpha=\operatorname{Exp}(D)$ be the corresponding automorphism. If one of the equations above holds for $\alpha$ and $\alpha$ has no non-trivial eigenvalues which are roots of unity, then the derivation $D$ is singular. ${ }^{1}$

Proof. Proof. Because $\operatorname{tr}(D)=0$, it follows that $\operatorname{det}(\operatorname{Exp} D)=e^{\operatorname{tr}(D)}=1$ so we can apply the theorem for $\operatorname{SL}(n, K)$. If $D$ is singular, then $D(v)=0$ for some $v \neq 0$. Hence $\operatorname{Exp} D(v)=v$. Since $\operatorname{Exp} D$ has $v$ as a fixed point and determinant 1 , one or the other of these two equations holds. Conversely, if equation 2 holds and therefore by Theorem $1 \operatorname{Exp} D(v)=v$ for some $v \neq 0$, then $\frac{\operatorname{Exp} D-I}{D}(D(v))=0$. By our assumption on the eigenvalues of $D$, it follows that $\frac{\operatorname{Exp} D-I}{D}$ is invertible and hence $D(v)=0$.

Now let $A \in M_{n}(\mathbb{R})=\mathcal{M}$ and have it act on $\mathcal{M}$ by $\operatorname{ad}_{A}(X)=[A, X]$. When $X$ is in the linear span of $I$ and $A, \operatorname{ad}_{A}(X)$ is automatically 0 . Here we shall be interested in non-trivial such $X{ }^{2}$

Taking the matrix units $E_{i, j}$ as a basis for $\mathcal{M}$ we see

$$
\operatorname{tr}\left(\operatorname{ad}_{A}\right)=\sum_{i=1}^{n} \operatorname{ad}_{A}\left(E_{i, i}\right)=\operatorname{ad}_{A}\left(\sum_{i=1}^{n} E_{i, i}\right)=\operatorname{ad}_{A}(I)=0 .
$$

Let

$$
\mathcal{X}=\{X \in \mathcal{M}: \operatorname{tr}(A X)=0\} .
$$

Then $\mathcal{X}$ is a subspace of $\mathcal{M}$ invariant under $\operatorname{ad}_{A}$. This is because $\operatorname{tr}(A[A, X])=$ $\operatorname{tr}\left(A^{2} X\right)-\operatorname{tr}(A X A)$ and since $\operatorname{tr}(Y Z)=\operatorname{tr}(Z Y)$ for all $Y$ and $Z$, this is zero (whether $X \in \mathcal{X}$, or not).

Assume $\operatorname{tr}\left(A^{2}\right) \neq 0$. Then

$$
\mathcal{A} \oplus \mathcal{X}=\mathcal{M}
$$

where $\mathcal{A}$ is the line through $A$. $\mathcal{A} \cap \mathcal{X}=(0)$ because if $\operatorname{tr}(A \lambda A)=0$, then $\lambda \operatorname{tr}\left(A^{2}\right)=0$ and so $\lambda=0$. The sum of these subspaces is $\mathcal{M}$ since if $X \in \mathcal{M}$, there is a real $\lambda$ with $\operatorname{tr}((X-\lambda A) A)=0$. For this we take

$$
\lambda=\frac{\operatorname{tr}(X A)}{\operatorname{tr}\left(A^{2}\right)} .
$$

\footnotetext{
${ }^{1}$ Since $\alpha$ has no eigenvalues which are roots of unity, a theorem of N. Jacobson tells us $\mathfrak{g}$ is actually nilpotent. See [2], Theorem 5.4.2.

${ }^{2}$ It is a pleasure for the author to acknowledge a number of useful conversations on this material with Laura Geatti.
} 
Choosing a basis for $\mathcal{X}$ and, by adding $A$, extending this to a basis of $\mathcal{M}$ we see

$$
\operatorname{ad}_{A}=\left(\begin{array}{ccc|c}
\operatorname{ad}_{A} \mid \mathcal{X} & & & 0 \\
& & & 0 \\
& & & 0 \\
\hline 0 & 0 & 0 & 0
\end{array}\right) .
$$

Hence $\operatorname{Spec}\left(\operatorname{ad}_{A}\right)=\operatorname{Spec}\left(\operatorname{ad}_{A} \mid \mathcal{X}\right) \cup(0)$ so since $\operatorname{tr}\left(\operatorname{ad}_{A}\right)=0, \operatorname{tr}\left(\operatorname{ad}_{A} \mid \mathcal{X}\right)$ is also 0. Moreover, exponentiating equation 3 we get,

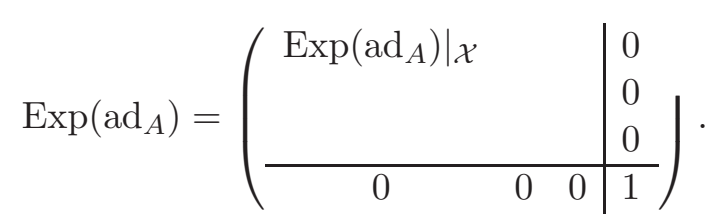

Now since $A$ has all real eigenvalues, it can be put in triangular form over $\mathbb{R}$. Hence $\operatorname{tr}\left(A^{2}\right)$ is the sum of the squares of its eigenvalues. This means $\operatorname{tr}\left(A^{2}\right)>0$ unless all eigenvalues are zero, i.e. $A$ is nilpotent.

We recall that $\operatorname{Exp} \operatorname{ad}_{A}=\operatorname{Ad}_{\operatorname{Exp}(A)}$ and first assume $A$ is not nilpotent. In [1] Section 3.3 an equivariant isomorphism $\phi$ is established,

$$
\phi: \mathcal{M} \rightarrow \mathbb{R}^{n} \otimes \mathbb{R}^{n}
$$

with $\operatorname{ad}_{A}=\phi^{-1}\left(A \otimes I-I \otimes A^{t}\right) \phi$. Because $\operatorname{tr}(A)=\operatorname{tr}\left(A^{t}\right)$, it follows that $\operatorname{tr}\left(\operatorname{ad}_{A}\right)=\operatorname{tr}(A) n-n \operatorname{tr}\left(A^{t}\right)=0$. Since $A$ has only real eigenvalues, the eigenvalues of $\operatorname{ad}_{A}$ acting on $\mathcal{M}$ being differences of the eigenvalues of $A$, are also real. Because $\mathcal{X}$ is invariant under $\operatorname{ad}_{A}$, the eigenvalues of its restrictions to $\mathcal{X}$

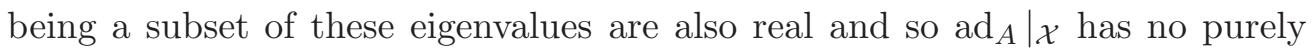
imaginary eigenvalues at all. Since $\operatorname{ad}_{A}$ has trace zero, it follows from equation $3, \operatorname{tr}\left(\operatorname{ad}_{A} \mid \mathcal{X}\right)=0$.

Moreover, from the Jordan form of $A$ one sees easily that in each Jordan block $\operatorname{Exp}(A)$ is a product of a scalar matrix and a unipotent matrix. Hence on each of these subspaces $\operatorname{Ad}_{\operatorname{Exp}(A)}$ acts (by conjugation) as a unipotent matrix. But the eigenvalues of this action by conjugation are quotients of the eigenvalues of this unipotent matrix. Hence all the eigenvalues on each block are all 1 and so $\operatorname{Ad}_{\operatorname{Exp}(A)}=\operatorname{Exp}\left(\operatorname{ad}_{A}\right)$ is itself unipotent. Equation 4 then tells us $\operatorname{Ad}_{\operatorname{Exp}(A)}\left|\mathcal{X}=\operatorname{Exp}\left(\operatorname{ad}_{A}\right)\right|_{\mathcal{X}}$ is unipotent as well. By the previous corollary there is a non-zero $X \in \mathcal{X}$ with $[A, X]=0$. On the other hand, if $A$ were nilpotent, since the eigenvalues of $\operatorname{ad}_{A}$ are differences of those of $A, \operatorname{ad}_{A}$ is a nilpotent operator on $\mathcal{M}$ and so $\mathrm{ad}_{A}^{j}=0$ for some integer $j \geq 1$. If $j \geq 2$, 
$\operatorname{ad}_{A}(X)=0$ for all $X \in \operatorname{ad}_{A}^{j-1}$. Thus $[A, X]=0$ for all these $X$. If $j=1$, then $[A, X]=0$ for all $X \in \mathcal{M}$.

This proves that if $A \in \mathcal{M}$ has only real eigenvalues and one of the following equations holds (according to whether $n$ is odd or even).

$$
\sum_{i=1}^{n^{2}-1}(-1)^{i-1} \sigma_{i}\left(\operatorname{Ad}_{\operatorname{Exp}(A)}\right)=0 \quad \sum_{i=1}^{n^{2}-1}(-1)^{i-1} \sigma_{i}\left(\operatorname{Ad}_{\operatorname{Exp}(A)}\right)=2,
$$

then $[A, X]=0$ for some $X \in \mathcal{M} \backslash \mathcal{A}$.

We now sharpen this result to obtain non trivial $X \in \operatorname{Ker}\left(\operatorname{ad}_{A}\right)$. For this we shall have to assume the eigenvalues of $A$ are not merely real, but actually non-negative.

Let $\mathcal{B}=$ lin.sp. $\mathbb{R}\{A, I\}$. If $A$ and $I$ are linearly dependent, the statement of Corollary 3 below follows from the above so we now assume $A$ and $I$ are linearly independent. Consider $I+\lambda_{*} A=Y_{*}$. We choose $\lambda_{*}$ so that $Y_{*} \in \mathcal{X} \cap \mathcal{B}$. Since this is in $\mathcal{B}$ for any $\lambda_{*}$, we need

$$
0=\operatorname{tr}\left(\left(I+\lambda_{*} A\right) A\right)=\operatorname{tr}(A)+\lambda_{*} \operatorname{tr}\left(A^{2}\right) .
$$

That is, $\lambda_{*}=-\operatorname{tr}(A) / \operatorname{tr}\left(A^{2}\right)$ and, as above, this can be done as long as $A$ is not nilpotent. Then $Y_{*} \in \mathcal{X} \cap \mathcal{B}$ and is non-zero since $A$ and $I$ are linearly independent. Call $\mathcal{Y}_{*}$ the 1 dimensional subspace of $\mathcal{X}$ generated by $Y_{*}$. Evidently, $\operatorname{ad}_{A}\left(Y_{*}\right)=0$ and in a similar manner to the above we will further decompose $\mathcal{X}$ into a direct sum of $\operatorname{ad}_{A}$-invariant spaces $\mathcal{X}=\mathcal{Y}_{*} \oplus \mathcal{X}_{*}$, where $\mathcal{X}_{*}=\{X \in \mathcal{X}: \operatorname{tr}(X)=0\}$. This is so since $\operatorname{tr}\left(X\left(I+\lambda_{*} A\right)\right)=0$ if and only if $\operatorname{tr}(X)+\lambda_{*} \operatorname{tr}(X A)=0$ and since $X \in \mathcal{X}, \operatorname{tr}(X A)=0$ automatically.

Now these spaces are disjoint because if $I+\lambda_{*} A$ had trace zero, then $n+$ $\lambda_{*} \operatorname{tr}(A)=0$ so $\lambda_{*}=-n / \operatorname{tr}(A)$ and hence

$$
n=\operatorname{tr}(A)^{2} / \operatorname{tr}\left(A^{2}\right),
$$

the denominator being positive since $A$ is not nilpotent. Taking $a_{i}$ to be the non-negative eigenvalues of $A$ and applying the Cauchy-Schwarz inequality to the vectors $\left(a_{1}, \ldots, a_{n}\right)$ and $(1, \ldots, 1)$, we see

$$
\sum_{i} a_{i} \leq \sqrt{n}\left(\sum_{i} a_{i}^{2}\right)^{1 / 2}
$$

But from equation 5 it follows that $\sum_{i} a_{i} \geq \sqrt{n}\left(\sum_{i} a_{i}^{2}\right)^{1 / 2}$. Hence these quantities are equal. By the case of equality of Cauchy-Schwarz it follows that all 
the $a_{i}$ are equal so $A$ is a scalar and hence $A$ and $I$ are linearly dependent, a contradiction. Thus we get a direct sum decomposition of $\operatorname{ad}_{A}$-invariant subspaces,

$$
\mathcal{M}=\mathcal{B} \oplus \mathcal{X}_{*}
$$

Since $\operatorname{ad}_{A} \mid \mathcal{X}_{*}$ must have trace zero and $\operatorname{Exp}\left(\operatorname{ad}_{A}\right)$ has all its eigenvalues equal to 1 on $\mathcal{X}$, the same is true when this operator is restricted to $\mathcal{X}_{*}$. Applying Corollary 2 (assuming $A$ is not nilpotent) we see

Corollary 3. Suppose $A \in \mathcal{M}$ has only non-negative eigenvalues and one of the following equations holds (according to whether $n$ is odd or even)

$$
\sum_{i=1}^{n^{2}-1}(-1)^{i-1} \sigma_{i}\left(\operatorname{Ad}_{\operatorname{Exp}(A)}\right)=0 \quad \sum_{i=1}^{n^{2}-1}(-1)^{i-1} \sigma_{i}\left(\operatorname{Ad}_{\operatorname{Exp}(A)}\right)=2 .
$$

Then $[A, X]=0$ for some $X \in \mathcal{M} \backslash \mathcal{B}$.

On the other hand if $A$ is nilpotent then, as above, $\operatorname{ad}_{A}$ is nilpotent and remains so when restricted to any invariant subspace. The argument then proceeds just as before.

Denote by $\mathfrak{t}(n, \mathbb{R})$ the real triangular matrices of order $n$ and $T(n, \mathbb{R})$ those with positive diagonal entries. We first observe

$$
\operatorname{Exp}: \mathfrak{t}(n, \mathbb{R}) \rightarrow T(n, \mathbb{R})
$$

is a global diffeomorphism because $T(n, \mathbb{R})$ is a connected and simply connected real solvable Lie group with all real Ad roots. Now let $A \in M_{n}(n, \mathbb{R})$ and assume it has all real eigenvalues. Then, as above, $A$ can be put in triangular form over $\mathbb{R}$ as can $\operatorname{Exp}(A)$, which has all its eigenvalues positive. Since $\operatorname{Ad}_{\operatorname{Exp}(A)}=\operatorname{Exp}\left(\operatorname{ad}_{A}\right)$ and Exp is global diffeomorphism, the action of conjugation by $\operatorname{Exp}(A)$ on $\mathcal{M}$ is equivariantly equivalent to the action of $\operatorname{ad}_{A}$ on $\mathcal{M}$. Now assume further that the eigenvalues of $A$ are actually non-negative. Then by Corollary 3 above there is an $X \in \mathcal{M} \backslash \mathcal{B}$ so that $[A, X]=0$. Therefore $X$ also commutes with $\operatorname{Exp}(A)$. This yields,

Corollary 4. Let $A \in \mathcal{M}$ and and have all non-negative eigenvalues. Then the action of $\operatorname{Exp}(A)$ on $\mathcal{M}$ by conjugation has a non-trivial fixed point. 


\section{References}

[1] H. Abbaspour, M. Moskowitz, Basic Lie Theory, World Scientific, Singapore (2007), doi: $10.1142 / 6462$.

[2] I. Farmakis, M. Moskowitz, Fixed Point Theorems and their Applications, World Scientific, Singapore (2013), doi: 10.1142/8748. 